\title{
CROSS CORRELATION ANALYSIS OF MULTI-CHANNEL NEAR INFRARED SPECTROSCOPY
}

\author{
Raul Fernandez Rojas ${ }^{1}, \mathrm{Xu} \mathrm{Huang}^{1}$, Keng Liang Ou ${ }^{2}$ and \\ Jehu Lopez-Aparicio ${ }^{3}$ \\ ${ }^{1}$ Faculty of Education, Science, Technology and Mathematics, \\ University of Canberra, Australia \\ \{raul.fernandezrojas, xu.huang\} @canberra.edu.au \\ ${ }^{2}$ College of Oral Medicine, Taipei Medical University, Taiwan \\ klou@tmu.edu.tw \\ ${ }^{3}$ Faculty of Sciences, National Autonomous University of Mexico, Mexico \\ jehudciencias.unam.mx
}

\begin{abstract}
In this paper we present the use of a signal processing technique to find dominant channels in near infrared spectroscopy (NIRS). Cross correlation is computed to compare measuring channels and identify delays among the channels. In addition, visual inspection was used to detect potential dominant channels. The results showed that the visual analysis exposed painrelated activations in the primary somatosensory cortex (S1) after stimulation which is consistent with similar studies and the cross correlation analysis found dominant channels on both cerebral hemispheres. The analysis also showed a relationship between dominant channels and neighbouring channels. Therefore, our results present a new method to detect dominant regions in the cerebral cortex using near-infrared spectroscopy. These results have also implications in the reduction of number of channels by eliminating irrelevant channels for the experiment.
\end{abstract}

\section{KEYWORDS}

Signal processing, functional response, time dependant, medical imaging analysis, fNIRS.

\section{INTRODUCTION}

Near-infrared spectroscopy (NIRS) is an optical topography technique that enables non-invasive continuous monitoring of local hemodynamic response associated with cortical activity. NIRS has been applied in studies to assess cerebral functioning (functional NIRS or fNIRS) such as tasks on motor skills [1], processing of faces [2, 3] and language skills development in infants [4, $5]$, or perception of pain [6,7]. However, the large number of channels $(24,48$, or 52) used to measure simultaneously the brain activity in patients makes the analysis and post processing of brain signals a demanding task. For that reason, region of interest (ROI) of NIRS signals are of high importance.

David C. Wyld et al. (Eds) : ITCS, JSE, SIP, ARIA, NLP - 2016

pp. 23-33, 2016. (C) CS \& IT-CSCP 2016

DOI : $10.5121 /$ csit.2016.60303 
In medical imaging, region of interest can be defined as a specific area (anatomical location) on a digital image that is designated to implement further examination. ROIs are of common use in functional magnetic resonance imaging (fMRI) where activated regions are of particular interest. ROIs are defined on the hypothesis that the information is retained in activity patters among groups of neurons[8] and that across different patients there are regions in the human brain that exhibit the same functionality[9]. Poldrack[10] provides three reasons to select specific regions for further analysis. The first reason to perform a ROI analysis is to detect activity patterns across different conditions or variables. The second reason is to decrease the number of statistical tests to control for Type I error. The third purpose is to limit testing to a smaller region that is selected on the basis of functionally specific regions such as retinotopically organized regions in primary visual cortex or motion-sensitive regions in middle temporal cortex. In fMRI ROIs are defined either in terms of specific structural or functional features[8], however the best practice is to define ROIs for each subject based on their own anatomy[10].

In functional near-infrared spectroscopy, regions of interest are also of use. ROI analysis can be done through analysis of channels (also called Channels of Interest or COI) which show a significant increase in the hemodynamic concentration after external stimulation compared to the base-line measurement. In the neurosciences field the changes in blood circulation by evoked stimulation is also known as "hemodynamic response"[11]. In the literature, there are diverse methods proposed to obtain region of interest in functional NIRS. For example, probabilistic mapping methods[12], contrast-to-noise-ratio (CNR)[13], principal component analysis (PCA)[5], or Cross-correlation analysis[7]. Nonetheless, these methods do not provide the dominant channel, and neither the relationship between the dominant channel and adjacent channels; these two features are important to reduce the number channels for further processing.

For these reasons, this paper proposes a technique to identify the dominant channel in each cerebral hemisphere by using cross correlation analysis. In this study, we use a data set of cerebral hemodynamic responses from an acupuncture stimulation test on six subjects [7]. The analyses showed the existence of dominant channels, the association with neighbouring channels and the extent of the activated area in the brain cortex.

\section{MATERIALS AND METHODS}

\subsection{Topography Equipment}

Data was obtained using the Hitachi ETG-4000 (Hitachi Medical Corporation) to investigate cerebral hemodynamics by NIRS. The measuring equipment utilises near infrared light to monitor cortical hemodynamics. NIR light is transmitted to the patient's head using multiple optical fibre emitters (Fig. 1, red circles). The near infrared light penetrates head tissue and bone to reach the cerebral cortex. Some NIR light is absorbed by hemoglobin, while the non-absorbed light is reflected to the source, where it is sampled by an optical detector (Fig. 1, blue circles). The NIR light between emitters and detectors is sampled at a given time point named channels (Fig. 1, numbered squares). Because the absorption of NIR light of Oxy-hemoglobin ( $\mathrm{HbO}$ ) and Deoxy-hemoglobin (HbR), two wavelengths of light $(695$ and $830 \mathrm{~nm})$ are needed for their continuous monitoring; while total hemoglobin $(\mathrm{HbT})$ is calculated as the difference between $\mathrm{HbO}$ and $\mathrm{HbR}$. The sample frequency used in this experiment was of 10 samples per second. The probe configuration used for this study was using two probes of 12 channels to measure neurologic activity. The measurement area examined is the bilateral motor cortex area, as we 
expected to obtain hemodynamic response in the somatosensory cortex area $(\mathrm{S} 1)[14,15]$. According to the international EEG 10-20 system[16], the probes were centred on the C3 and C4 position. Fig. 1 shows the 24-channel configuration used in the study, channels 1 to 12 represent the right hemisphere, while channels 13 to 24 represent the left hemisphere.

\subsection{Participants and Experimental Protocol}

Six healthy right-handed subjects ( 2 females, 4 males) were examined in the experiments, age range 25 to 35 years (mean age 27.8) old were included. Written consent was obtained from all participants prior to initiation of the experiments. Subjects with a history of a significant medical disorder, a current unstable medical condition, or currently taking any medication, were excluded. All participants were explained about the acupuncture procedure and manipulations, and the subjects had the opportunity to stop the procedure at any time if needed. In addition, all the subjects reported that never had used any type of acupuncture treatment in the past.
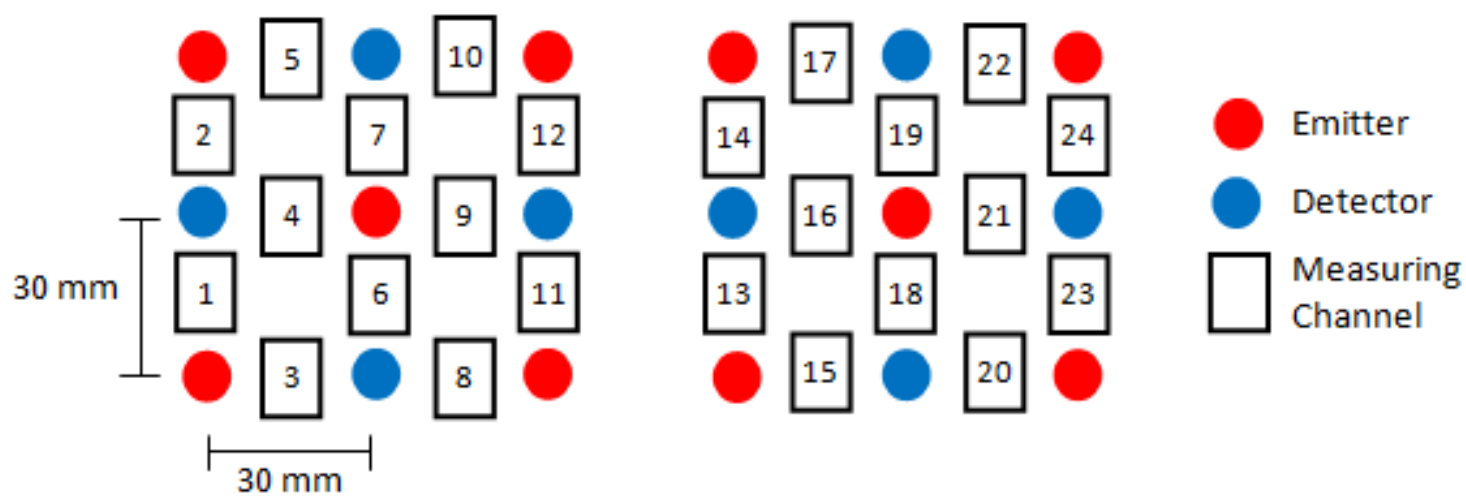

Figure 1. Channel configuration, right hemisphere (channels 1-12) and left hemisphere (channels 13-24).

The experiments were designed by the School of Oral Medicine of Taipei Medical University (TMU, Taiwan) in collaboration with the University Of Canberra (UC, Australia). The study and methods were carried out in accordance with the guidelines of the declaration of Helsinki and approved by full-board review process of the TMU-Joint Institutional Review Board under contract number 201307010. All experiments were carried out at TMU in a quiet, temperature (22-24o) and humidity (40-50\%) controlled laboratory room. The experiments were done in the morning (10:00am-12:00pm) and each experiment lasted around 30 minutes. Quantitative data was collected using the ETG-4000 with the patients sat down in an ergonomic chair near the topography system (Fig. 2, left panel).

In order to obtain stimulation-related activation in the cerebral cortex, acupuncture was used to induce pain stimulation in a safe manner. Brand new acupuncture needles were used for each experiment, and using traditional Chinese acupuncture techniques that were performed by an acupuncturist of TMU Hospital. The puncture point used for stimulation was the "Hegu Point", located on top of the hand, between the thumb and forefinger (Fig. 2, right panel). This point was used because it is an area of easy access and the hand can be set aside while the patient is relaxed on the chair. Each patient was punctured on both hands, each hand on separate days; each hand was treated as a separated experiment[7]. The acupuncture procedure consisted of three types of acupuncture stimulations (tasks). Each task lasted 5 seconds and after each stimulation a resting time (Rt) of 30 seconds was done. The first stimulation is needle insertion (T1), the three 
following stimulations are needle twirl to increase Qi (T2), and the last stimulation is needle removal (T3). The complete data set was used as primary source to assess our dominant channel identification method.

\subsection{Time-Dependant Cross Correlation}

In signal processing, cross correlation is used to calculate the temporal similarity between two signals. In the NIRS context, cross correlation is employed to compare NIRS signals and to identify the dominant channels on both hemispheres. The cross correlation function was computed between channels 1-12 in the right probe and 13-24 in the left probe. This measure of temporal similarity of two signals can be done by computing a time-shifting along one of the input signals. The cross correlation between two waveforms $x(t)$ and $y(t)$ can be defined as follows: $r_{x y}(\tau)=\sum_{-\infty}^{\infty} x(t) y(t-\tau)$. Where $\tau$ is the time-lag between $x(t)$ and $y(t)$, the value of $r_{x y}$ denotes the difference (lag/lead) between channel signal $y(t)$ and channel signal $x(t)$. The cross correlation value between two channels in the same probe is done after each stimulation from $-40 \mathrm{sec}$ to $+40 \mathrm{sec}$ at a rate of 10 samples per second.

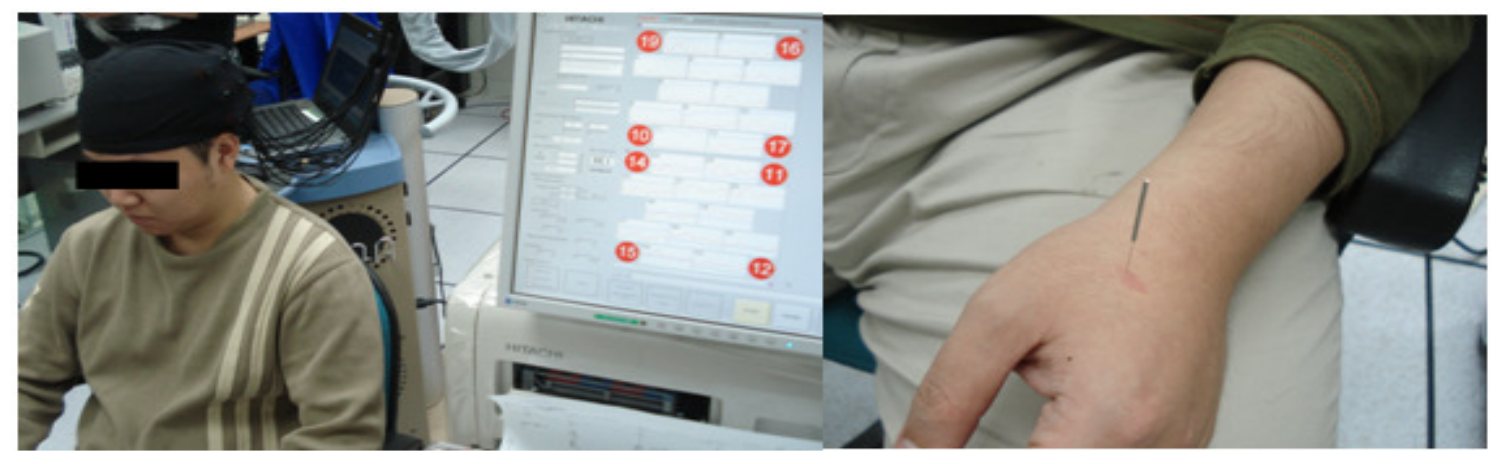

Figure 2. Left image shows a subject wearing the multi-channel probe next to the spectroscopy equipment. Right image presents the acupuncture needle in the "Hegu point".

\subsection{Evaluation Approach}

To evaluate our experiment, visual inspection can be done on activation patterns in both hemispheres and will be focus on locating potential dominant areas. The visual analysis will be carried out by configuring the NIRS data following the channel configuration shown in Fig 1 . This analysis will also provide evidence of cortical activity that is associated with the stimulation-related sensory processing in the brain cortex.

\section{RESULTS}

\subsection{Visual Pattern Identification}

It was possible to depict activated regions in the NIRS data by visual inspection. Fig. 3 presents the colour representation of three sequential images from the right (Ch1-Ch12) and left (Ch13Ch24) hemispheres of subject four (male, 26 year old); only needle insertion task (T1) is presented in this study because it was the strongest stimulation reported by all subjects. The images were taken every five seconds after external stimulation, subject and sampling time were 
chosen for graphic purposes. Two events were observed, the existence of a dominant area and the propagation delay of activated areas in the brain cortex. These findings were consistent in all participants and on both hemispheres.

In the identification of dominant areas two situations can be noticed, the single-dominant region and the multi-dominant region. In the first case, the single-dominant region is when the dominant area is very evident and stands out among other regions. An example of this can be seen in Fig. $3 \mathrm{~A}$, the area around $\mathrm{Ch} 7$ (dotted oval) is very evident that this region has a higher response to external stimulus as compared to other areas. The second case is when the data have a multidominant response to evoked pain. Fig. 3B shows an example of this case, two main regions can be observed; the brain response is greater around $\mathrm{Ch} 19$ and Ch18. In this case the selection of a dominant channel is not as evident as the single-region case. In both cases, the selection of a dominant channel is made by visual perception; however in the multi-dominant response, another method is needed to identify the dominant channel.

After the initial visual analysis, a delay between the dominant area and surrounding areas was also eminent. This is evident in Fig. 3A, around $\mathrm{Ch} 7$ the magnitude of $\mathrm{HbO}$ traces is higher and seems to be the centre of propagation towards adjacent channels in posterior images. For example, in Fig. 3A-I, the area around Ch7 appears to be slightly more active than the rest of the channels. However, after five seconds (Fig. 3A-II) the activation has increased and reached Ch5 and Ch10. In the last image (Fig. 3A-III), the activation is stronger than previous image and radiation pattern expanded to neighbouring channels $\mathrm{Ch} 2, \mathrm{Ch} 4, \mathrm{Ch} 9$, and $\mathrm{Ch} 12$. This phenomenon suggests a strong relationship between $\mathrm{Ch} 7$ and surrounding channels.

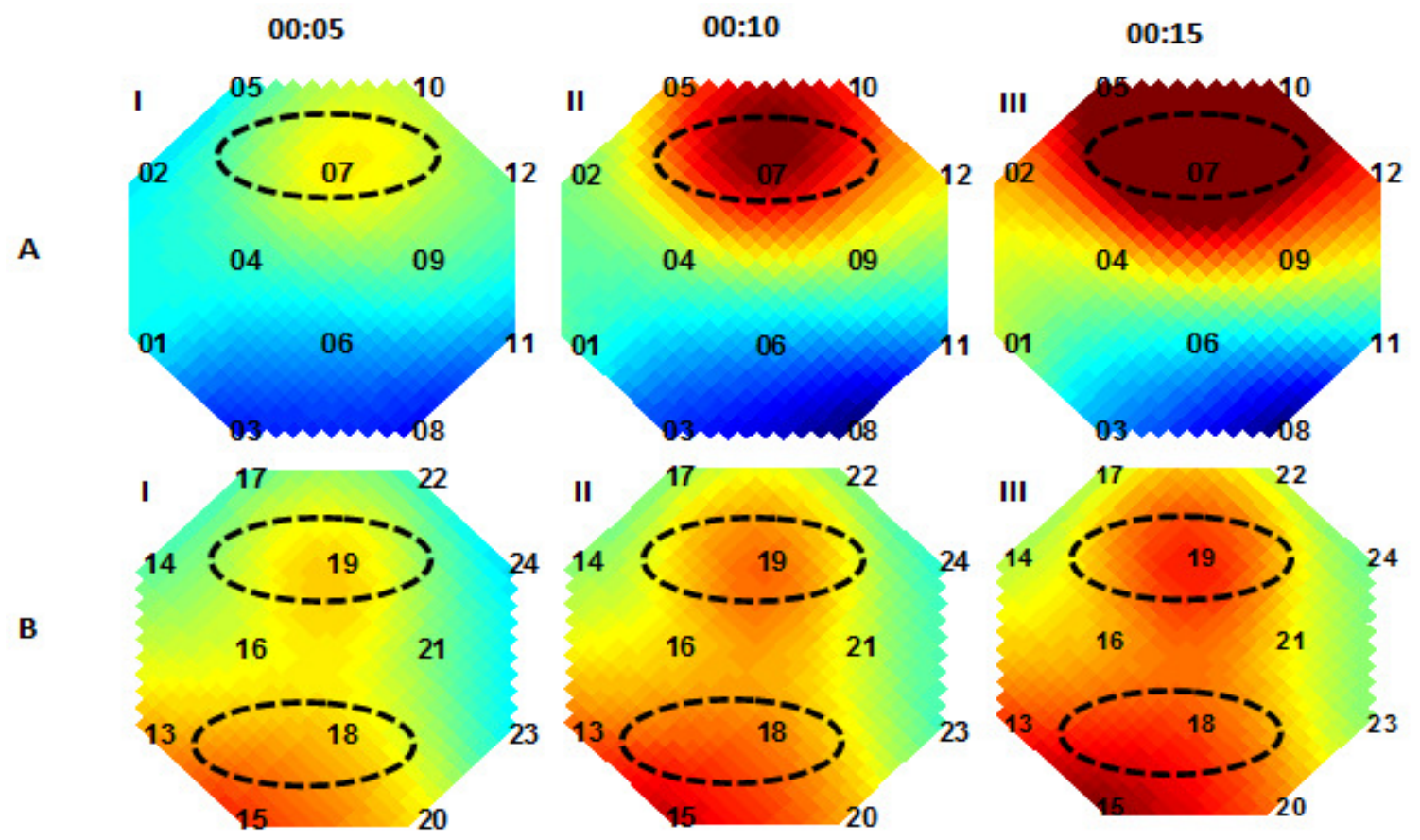

Figure 3. Visual analysis. A) Single dominant pattern. B) Multi-dominant pattern. Three images were taken every five seconds after stimulation. Dotted circles show the dominant region. Cortical activity was initially started in a small region and extended to surrounding areas. 


\subsection{Cross Correlation Signal Processing}

Based on the assumption that a dominant channel and a relationship among the channels exist, the cross correlation analysis was computed to examine these two ideas. Fig. 4 shows the results of two cross correlation comparisons of two channels, Ch7 (Fig. 4A) and Ch16 (Fig. 4B) on right and left cerebral hemispheres respectively, from subject four after the acupuncture stimulus.

Detecting the dominant channel in dominant regions was done by comparing the lags between potential dominant channel and the rest of the channels. The detection was carried out by correlating the delay times between possible dominant channel and the rest of channels in that hemisphere. Fig. 4A shows the cross correlation analysis and confirmed that the region around $\mathrm{Ch} 7$ was the dominant area. It was also found that $\mathrm{Ch} 5$, and Ch10 have no time delay $(\tau=0.0 \mathrm{sec})$ with Ch7, which suggests that these three channels are positioned over a potential region of interest due to the strong activation after stimulation. Moreover, cross correlation analysis of the opposite hemisphere (Fig. 4B) clarified the impression of having two dominant regions after visual analysis. This result illustrated that Ch16 lead the rest of the channels for this particular subject and experiment. It was also found that Ch18 $(\tau=+0.8 \mathrm{sec})$ and Ch19 $(\tau=+0.3 \mathrm{sec})$ have minimum lags against Ch16, which rejects the idea of having two separate dominant areas with high response to the external stimulation. Therefore, we can tell that for subject $4(n=4)$, Ch16 is the dominant channel on the left hemisphere. As a result, it is possible to tell that the dominant channel is the measuring channel with the fastest answer after external stimulation; and in most of the cases within this experiment, the dominant channel is the channel with the strongest $\mathrm{HbO}$ response after noxious stimulation.
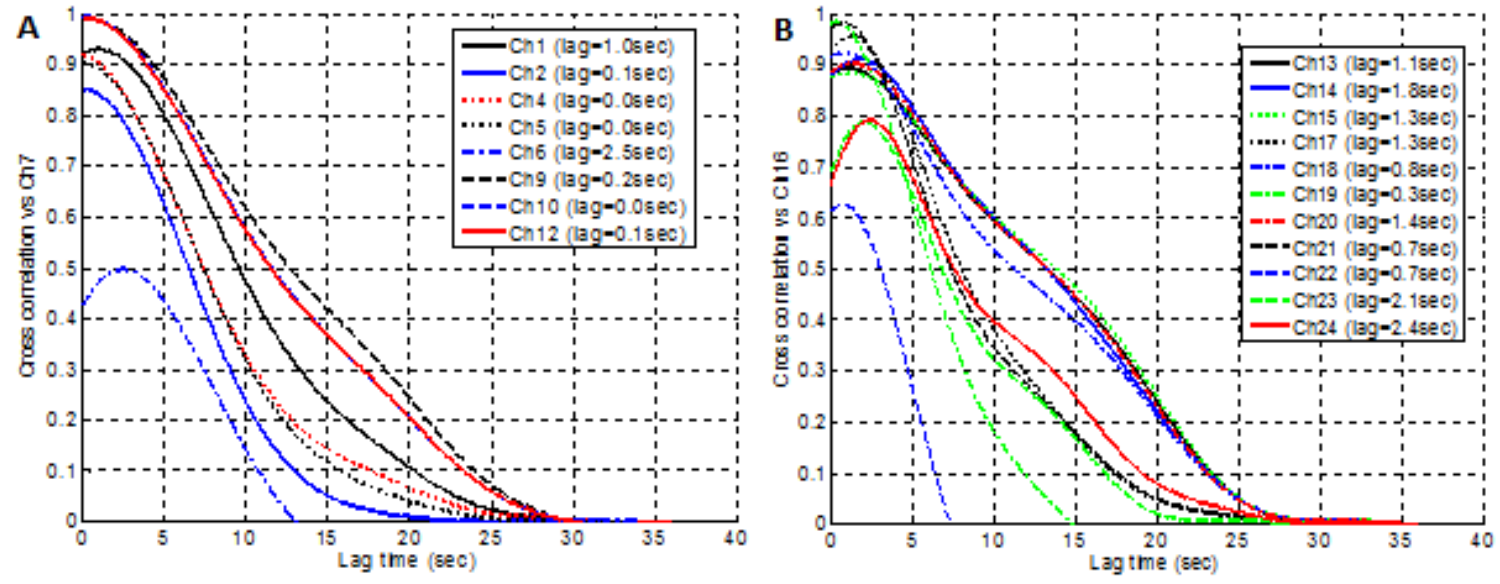

Figure 4. Cross correlation comparison of dominant channel and surrounding channels from subject four. A) Time-dependant evaluation of $\mathrm{Ch} 7$ and neighbouring channels. B) Time-dependant comparison between Ch16 and neighbouring channels.

Cross correlation signal processing was also used to determine the relationship between dominant channels and neighbouring channels. For instance, the results from subject four $(n=4)$ showed that $\mathrm{Ch} 7$ and surrounding channels have a small lag time that confirms that NIRS signals in dominant regions travel to surrounding regions. Fig. 5 presents the delays between channels on both hemispheres, we can see (Fig.5A) that region formed by Ch2, Ch4, Ch5, Ch7, Ch9, Ch10 and $\mathrm{C} 12$ has near cero delay $(\tau \approx 0 \mathrm{sec})$, while opposite channels Ch1, Ch3, Ch6, Ch8, Ch11 present a bigger delay against $\mathrm{Ch} 7$. This propagation is a clear indication of the relationship of 
NIRS signals between dominant channel and neighbouring channels. We can also see that channels (red squares) with small delays with dominant channel present a higher activation, while channels with larger delays belong to areas with limited activation (blue squares). This can be observed similarly in the left hemisphere as showed in Fig. 5B.
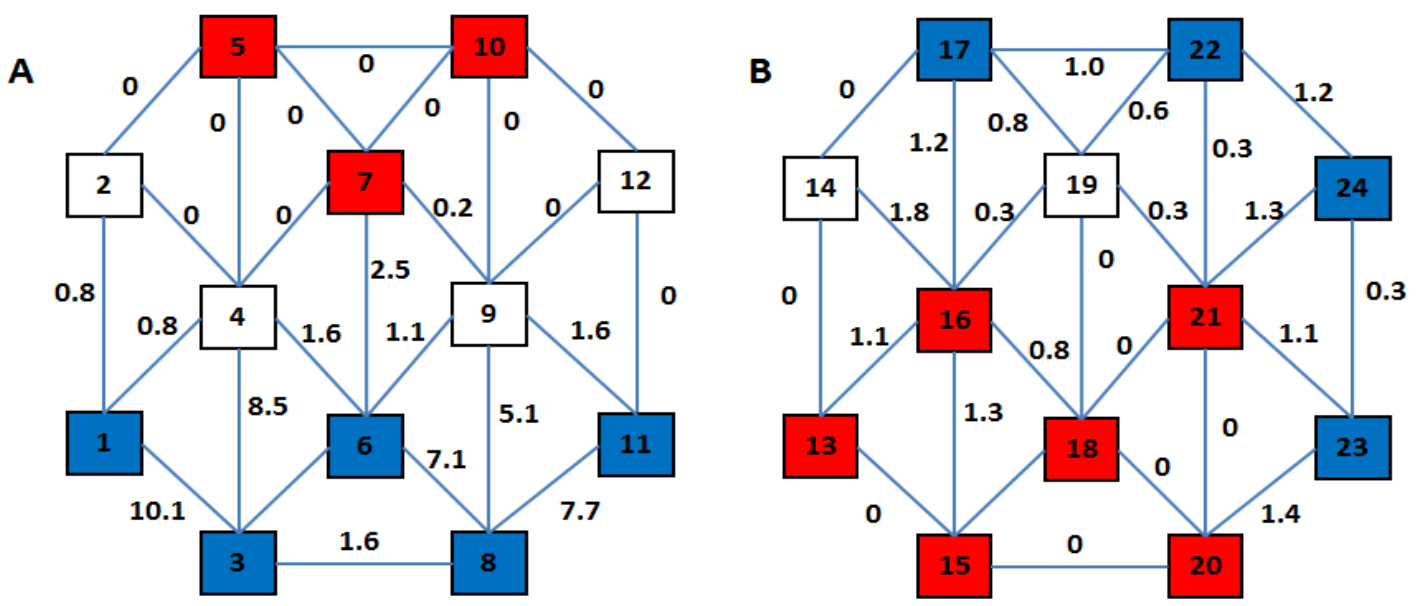

Figure 5. Cross correlation signal processing. Propagation time between activated areas (red) and less active channels (blue). A) right hemisphere. B) left hemisphere.

These results suggest that brain hemodynamic signals have a progressive movement from dominant regions towards peripheral regions. It is also important to note that the results after following stimulations (tasks) remained very similar, however, the lag and lead times between channels were slightly smaller. This situation can be explained by the fact that the first stimulation produced the strongest response in all the acupuncture experiment.

\section{DISCUSSION}

In the present study, we have used cross correlation signal processing to identify dominant channels in functional near-infrared spectroscopy (fNIRS). Cross correlation analysis finds the dominant region based on the lag times between channels and also provides an estimation of the relationship between channels. The visual inspection also corroborated the propagation effect and dominant patterns in the NIRS data.

We first used visual inspection of the stimulation-related activation patterns in the brain cortex to obtain an initial assessment of dominant channels. It was expected to obtain activation in functional areas where the cortical representation of pain is involved. It was noted that the brain response increased around the area of channel 7 ( $\mathrm{Ch} 7)$ on the right hemisphere and the area of channel 19 (Ch19) on the left hemisphere in all the subjects; being these two areas part of the postcentral gyrus in the parietal lobe. The postcentral gyrus is the location of the primary somatosensory cortex (S1), area that has been reported to be involved with the perception and modulation of painful somatosensory sensations[15]. It is also important to note that the cortical activation following the acupuncture stimulation was obtained on both hemispheres (bilateral S1 activation). These findings are in line with previous results $[15,17]$. However, other studies in pain stimulation have reported that pain activation can be also detected in the secondary somatosensory cortex (S2), anterior cingulate cortex (ACC), and insular cortex (IC)[14, 18, 19]. 
Based on this preliminary analysis, we expected to find ROIs in these areas and also confirmed the validity of our overall acupuncture experiment.

Cross correlation between channels was calculated to identify dominant channels and the relationship among channels. The analysis exhibited the existence of delays between channels, not only in the region of interest but also in the whole measuring probe. These delays between signals indicate that leading signals are dominant channels and based on the delays between channels, it is possible to say that the delays represent the movement of activated areas to surrounding areas. Moreover, our results showed that the dominant channel is one of the channels with the highest response to external stimulation.

An important result from the cross correlation analysis is that the number of control signals can be reduced by eliminating irrelevant channels. For example, channels with very small activation after stimulus can be excluded from the data base since they fail to represent any interaction within the experiment. Therefore, signal processing techniques such as cross correlation are of importance to find relevant channels that truly represent activations after stimulus.

\section{CONCLUSiOnS}

In conclusion, this study presents an analysis of near infrared spectroscopy (NIRS) signals by cross correlation. The cross correlation analysis exhibited the dominant channels in the region of interest on both hemispheres, and found that is the channel with the fastest response to external stimuli and with a very strong activation after stimulus. The results also showed that the time lags of the hemoglobin signals represent a relationship between dominant channel and neighbouring channels; these delays can be seen as the delay of localized cerebral blood flow from dominant areas traveling to surrounding areas. In addition, after a visual inspection, it was found that the acupuncture stimulation resulted in bilateral activation in the primary somatosensory region (S1) in all subjects. Based on the evaluations, our analysis successfully showed the region of interests and visualizes them as the apparent motion of cortical activity from dominant channel. Therefore, our results present a new method to detect region of interest in functional near-infrared spectroscopy.

\section{REFERENCES}

[1] Hatakenaka M, Miyai I, Mihara M, Sakoda S, Kubota K. Frontal regions involved in learning of motor skill—a functional NIRS study. NeuroImage. 2007;34:109-16.

[2] Kobayashi M, Otsuka Y, Nakato E, Kanazawa S, Yamaguchi MK, Kakigi R. Do infants represent the face in a viewpoint-invariant manner? Neural adaptation study as measured by near-infrared spectroscopy. Front Hum Neurosci. 2011;5.

[3] Honda Y, Nakato E, Otsuka Y, Kanazawa S, Kojima S, Yamaguchi MK, et al. How do infants perceive scrambled face?: A near-infrared spectroscopic study. Brain Res. 2010;1308:137-46.

[4] Gervain J, Macagno F, Cogoi S, Peña M, Mehler J. The neonate brain detects speech structure. Proc Natl Acad Sci USA. 2008;105:14222-7. 
[5] Kovelman I, Shalinsky MH, Berens MS, Petitto L-A. Shining new light on the brain's "bilingual signature": a functional Near Infrared Spectroscopy investigation of semantic processing. NeuroImage. 2008;39:1457-71.

[6] Bartocci M, Bergqvist LL, Lagercrantz H, Anand K. Pain activates cortical areas in the preterm newborn brain. Pain. 2006;122:109-17.

[7] Rojas F.R, Huang X, Ou KL, Tran D, Islam SMR. Analysis of pain hemodynamic response using near-infrared spsctroscopy (NIRS). Int J Mult Appl. 2015;7:31-42.

[8] Etzel JA, Gazzola V, Keysers C. An introduction to anatomical ROI-based fMRI classification analysis. Brain Res. 2009;1282:114-25.

[9] Nieto-Castanon A, Ghosh SS, Tourville JA, Guenther FH. Region of interest based analysis of functional imaging data. NeuroImage. 2003;19:1303-16.

[10] Poldrack RA. Region of interest analysis for fMRI. Soc Cogn Affect Neurosci. 2007;2:67-70.

[11] Ortiz E. Use of Neuroimaging to Clarify How Human Brains Perform Mental Calculations.Online Submission. 2010.

[12] Verner M, Herrmann MJ, Troche SJ, Roebers CM, Rammsayer TH. Cortical oxygen consumption in mental arithmetic as a function of task difficulty: a near-infrared spectroscopy approach. Front Hum Neurosci. 2013;7.

[13] Song X, Pogue BW, Jiang S, Doyley MM, Dehghani H, Tosteson TD, et al. Automated region detection based on the contrast-to-noise ratio in near-infrared tomography. Appl Opt. 2004;43:105362 .

[14] Hofbauer RK, Rainville P, Duncan GH, Bushnell MC. Cortical representation of the sensory dimension of pain. J Neurophysiol. 2001;86:402-11.

[15] Bushnell M, Duncan G, Hofbauer R, Ha B, Chen J-I, Carrier B. Pain perception: is there a role for primary somatosensory cortex? Proc Natl Acad Sci USA. 1999;96:7705-9.

[16] Homan RW, Herman J, Purdy P. Cerebral location of international 10-20 system electrode placement. Electroencephalogr Clin Neurophysiol. 1987;66:376-82.

[17] Sutherland MT, Tang AC. Reliable detection of bilateral activation in human primary somatosensory cortex by unilateral median nerve stimulation. NeuroImage. 2006;33:1042-54.

[18] Coghill RC, Sang CN, Maisog JM, Iadarola MJ. Pain intensity processing within the human brain: a bilateral, distributed mechanism. J Neurophysiol. 1999;82:1934-43.

[19] Apkarian AV, Bushnell MC, Treede RD, Zubieta JK. Human brain mechanisms of pain perception and regulation in health and disease. Eur J Pain. 2005;9:463-. 


\section{AUTHORS}

Raul Fernandez Rojas received his B. Eng. (Electronics) at the Universidad Tecnologica de la Mixteca, Mexico and his M. Eng. (Hons) at The Australian National University, Australia. Raul's research interests are robotics, computer vision, medical imaging, and signal processing.

$\mathrm{He}$ is currently pursuing a $\mathrm{PhD}$ degree in Information Sciences and Engineering at the University of Canberra, Australia.

Xu Huang received his B.E. and M.E. degrees and first Ph.D. in Electrical Engineering and Optical Engineering prior to 1989 and his second Ph.D. in Experimental Physics in the University of New South Wales, Australia in 1992. He has earned the Graduate Certificate in Higher Education in 2004 at the University of Canberra, Australia. Prof Xu's fields of research includes: cybersecurity, network security, Internet of Things (IoT), wireless and optical communications, cloud computing, digital signal processing, bio signal processing, brain computer interface (BCI), intelligent system, and smart networks.

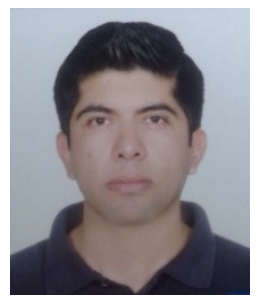

He has worked at the Australia National University from 1988 to 1990, the University of New South Wales from 1990 to 1995, also University of New England from 1995 to 2001. He is currently the Head of the Engineering at the Faculty of Information Sciences and Engineering, at the University of Canberra, Australia. He has been the Chair, Co-Chair, and TCM at various high quality International Conferences, and Editor for various high quality Journals. He has edited seven books, nine Book Chapters, 45 Journal Articles, and more than two hundred papers in high level of the IEEE and other international conferences (within ERA ranking); he has been awarded 17 patents in Australia in 2010 and 2013.

Prof. Huang has been a senior member of IEEE in Electronics and Computer Society since 1989, a Fellow in the Institute of Engineering Australian (FIEAust), Chartered Professional Engineering (CPEng), a Member of Australian Institute of Physics. He has been a member of Committee of the Institution of Engineering Australia at Canberra Branch for last 10 years.

Keng-Liang Ou graduated from the Mechanical Engineering Ph.D. program at the National Chiao Tung University, Taiwan. Professor Ou focuses his research on Biomaterials, Bioengineering, Biomedical Devices and Nanotechnology.

He went to Taipei Medical University to work on biomaterials research and development, and he is the current elected Dean of College of Oral Medicine in Taipei Medical University, Taiwan. He is also in charge of the Graduate Institute of Biomedical Materials and Tissue Engineering, Research Center for Biomedical Implants and Microsurgery Devices and Research Center for Biomedical Devices and

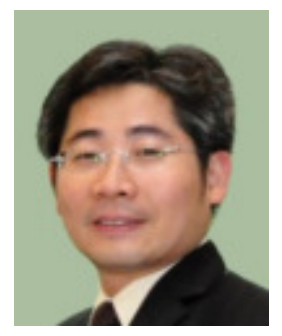
Prototyping Production.

Professor Ou is the President of Institute of Plasma Engineering Taiwan, the leader of The Taiwan society for metal heat treatment and the Head Taiwan Oral Biomedical Engineering Association. He is the leader and organizer for the biomedical product design, production, manufacturing, testing, legalization and market planning, with supports from team of scientists and researchers with different expertise. Professor Ou has extensive collaborations with industry and has played a major role in developing medical devices for health service professions in the world. Professor Ou was honorably awarded with the 49th Ten Outstanding Young Persons of Taiwan on 2011. 
Jehu Lopez-Aparicio received his Master degree in Electronics Sciences from the Meritorious Autonomous University of Puebla, Mexico in 2013. Jehu is currently completing his $\mathrm{PhD}$ at The National Autonomous University of Mexico. Jehu's research interests are: microelectronics design, biosensors design, nanoelectronics, and quantum physics.

He has been working at The National Autonomous University of Mexico since 2014 as an academic and electronics laboratory demonstrator. Jehu has also worked for CIDEC/Delphi technical center as a system engineer for two years.

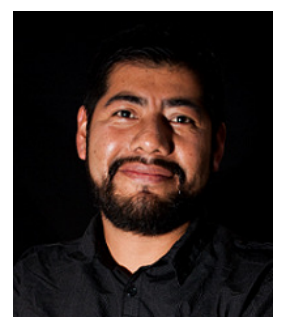

Mr Lopez-Aparicio has been awarded a patent for an electronic system to count photons, a project were he designed this system to complete his Master degree at the Meritorious Autonomous University of Puebla. Recently, Jehu also co-invented a biosensor to monitor in real-time glucose and insulin through a blood sample. This biosensor was awarded with the 2015 Latin America Google Research Award. 